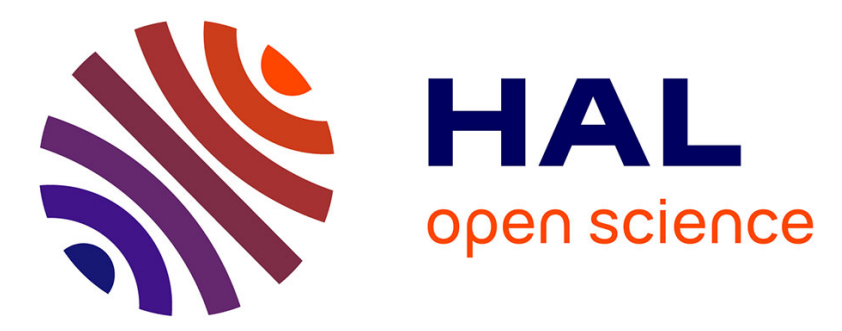

\title{
Proton Exchange Membrane Fuel Cell Prognosis Based on Frequency-Domain Kalman Filter
}

\author{
Yunjin Ao, Salah Laghrouche, Daniel Depernet, Kui Chen
}

\section{To cite this version:}

Yunjin Ao, Salah Laghrouche, Daniel Depernet, Kui Chen. Proton Exchange Membrane Fuel Cell Prognosis Based on Frequency-Domain Kalman Filter. IEEE Transactions on Transportation Electrification, 2021, 7 (4), pp.2332 - 2343. hal-03549355

\section{HAL Id: hal-03549355 https://hal.science/hal-03549355}

Submitted on 31 Jan 2022

HAL is a multi-disciplinary open access archive for the deposit and dissemination of scientific research documents, whether they are published or not. The documents may come from teaching and research institutions in France or abroad, or from public or private research centers.
L'archive ouverte pluridisciplinaire HAL, est destinée au dépôt et à la diffusion de documents scientifiques de niveau recherche, publiés ou non, émanant des établissements d'enseignement et de recherche français ou étrangers, des laboratoires publics ou privés. 


\title{
Proton exchange membrane fuel cell prognosis based on frequency domain Kalman filter
}

\author{
Yunjin Ao, Student Member, Salah Laghrouche, Daniel Depernet, and Kui Chen
}

\begin{abstract}
The degradation seriously affects the durability and cost of the Proton Exchange Membrane Fuel Cell (PEMFC). This paper presents a novel model-driven method based on the Frequency Domain Kalman Filter (FDKF) and voltage degradation model to predict the degradation of PEMFC in the frequency domain. The advantage of the proposed FDKF method is that it can process the data in groups; thus the computation time can be greatly reduced with high accuracy. Two degradation experiments under constant and quasi-dynamic currents have been used to demonstrate its prognosis performances under different conditions and different training times. Compared with the traditional time domain Extended Kalman Filter method and literature, it has been demonstrated that the proposed one has higher accuracy and requires much less calculation time.
\end{abstract}

Index Terms-Proton exchange membrane fuel cell, frequency domain Kalman filter, voltage degradation model, degradation prognosis, model-driven method

\section{INTRODUCTION}

A $\mathrm{S}$ fossil fuel is finitely reserved and it brings severe pollution to the environment, developing renewable energy is urgent [1]. Hydrogen fuel cells are promising alternatives as they use renewable sources and only discharge water, which is environment-friendly [2], [3]. Among all kinds of fuel cells, Proton Exchange Membrane Fuel Cell (PEMFC) is one of the most developed and concerned technologies, because of its lower pressure and ability to operate under normal temperature [4], [5].

PEMFCs have been applied to various fields, such as distributed power stations, mobile devices, and automobiles. For example, several manufacturers such as Peugeot, Toyota, and Benz have developed a fuel cell or fuel cell/electricity hybrid vehicles, such as Mirai and Citaro [6], [7]. However, the high cost and short lifetime remain the biggest challenges on the large-scale commercialization [8].

During the operation, the performance of PEMFC declines irreversibly, which is called degradation [9]. It includes the degradation of different components such as bipolar, catalyst, membrane, and electrode, thus it is a complex multi-physics, multi-units, and interactive process [10]. A lot of research works have been carried out by experiments or theoretical analysis, and some degradation mechanisms have been proposed. However, it is still a tricky task to fully understand and model it.

Yunjin Ao, Salah Laghrouche, Daniel Depernet and Kui Chen are with the Energy Department, FEMTO-ST Institute (UMR 6174), French National Centre for Scientific Research (CNRS), and FCLAB, FR CNRS 3539, Université Bourgogne Franche-Comté, Belfort/UTBM, Belfort 90400, France (e-mail:yunjin.ao@utbm.fr; salah.laghrouche@utbm.fr; daniel.depernet@utbm.fr; kui.chen@utbm.fr).

Manuscript received October 26, 2020.
To handle the problem of short lifetime, Prognostics and Health Management (PHM) are proposed to predict the degradation process and get the estimation of Remaining Useful Life (RUL) of PEMFC [11]. Based on PHM, certain measures can be taken to prevent degradation and improve lifetime [12]. The existing prognosis methods can be divided into three categories: model-driven methods, data-driven methods, and hybrid methods.

The model-driven methods learn and predict the PEMFC degradation trend based on specific PEMFC degradation models. The degradation models can be divided into three categories, i.e., physical models, empirical models, and semiempirical models [10], [13]. Mathieu Bressel et al. predicted the degradation and RUL of a PEMFC based on the Extended Kalman Filter (EKF) and a new empirical model, and the uncertainty of the prediction was quantified [12], [14]. At the same time, a particle filter and a voltage degradation model were applied by Marine Jouin et al., and the degradation and RUL were predicted [15]. Also, a model that considered the characterization disturbances and voltage recovery was researched by them [16]. Meanwhile, Kui Chen et al. researched the voltage degradation by unscented Kalman filter, and three different models were tried. The result was validated by three PEMFCs in different Fuel Cell Electric Vehicle (FCEV) with real load mission [17]. Model-driven methods can give explicit expressions of degradation. However, as the degradation is highly nonlinear, it is not easy to find a suitable model that can be widely used for different fuel cells [9].

The data-driven methods use the historic operation data to learn the inner relationship between them and then predict the degradation. Those methods need no specific models, and they are realized by artificial intelligence [18], [19]. In Ref. [20] [21] [22], Echo State Network (ESN) was used to forecast the aging process, and the most influential parameters of ESN were analyzed by analysis of variance method. Ma Rui et al. studied 8 experiments on 3 different PEMFCs by grid long short-term memory recurrent neural network, and the result corresponded well with experiments [23]. At the same time, in Ref. [24], the degradation of the real mission FCEV was predicted by a combined method which was based on the wavelet analysis, extreme learning machine, and genetic algorithm. In Ref. [25], the adaptive neuro-fuzzy inference system method was given in detail, and the parameters of the method were researched. Generally, these data-driven methods need enough amount of data to recognize the hidden relationship between them, and there are more parameters that need to be decided, than model-driven methods. Also, those parameters have little physical meanings; thus it is difficult to identify the causality 
based on the data-driven methods [10].

The hybrid methods are the combination of model-driven methods and data-driven methods. In Ref. [26], a hybrid prognostic method was proposed, which was based on the least square support vector machine and regularized particle filter. Meanwhile, Zhou Daming et al. combined Nonlinear Autoregressive Neural Network (NANN) and Particle Filter $(\mathrm{PF})$, as NANN was good at local fluctuation prediction while $\mathrm{PF}$ could give long-term degradation trends [27]. The hybrid methods can combine the advantages of both model-driven and data-driven methods, but as more than two methods are connected, the complexity of the hybrid methods is usually higher than a single model-driven or data-driven method [28].

By the analysis above, it can be seen that all those methods have their advantages and disadvantages. Usually, the modeldriven methods can give the long-term trend with less computation, and the explicit relationship can help with decisionmaking. However, the existing model-driven methods are still time-consuming when there is a lot of data to process, thus any reduction in the computation complexity is advantageous [29]. Therefore, a novel method based on FDKF is proposed in this paper, which can achieve accurate prediction and which is faster than traditional methods.

In this paper, we contribute to PEMFC prognosis by introducing a new model-driven method based on Frequency Domain Kalman Filter (FDKF), which can achieve voltage degradation prediction quickly and accurately. FDKF is initially used on Acoustic Echo Cancellation (AEC) problem [30], [31], and it is adapted to the PEMFC prognosis field for the first time. The prognosis process is achieved in the frequency domain. Four different voltage degradation models are researched by the proposed method, and the results are compared with each other. Different training times are also researched, which proves that the method is robust for both short-term and long-term prediction. The method is validated by two groups of experimental data, which were obtained under constant and quasi-dynamic current respectively. The main contributions of this paper are as follows:

(1) A new model-driven prognosis method is proposed for PEMFC degradation prediction, which is accurate and efficient. The advantage of the proposed FDKF method is that it can process data in groups, thus the computation time can be greatly reduced.

(2) Four different PEMFC voltage degradation models are studied with the proposed FDKF method. Linear model, quadratic model, logarithmic model, and exponential model are employed and compared under the framework of the FDKF method.

(3) Two groups of experimental data are used to validate the proposed method. One of the experiments was carried out under a constant current and the other under quasi-dynamic current. The degradation of PEMFC under different conditions can be predicted by the proposed method, which proves that the method is robust.

(4) The proposed FDKF method is compared with the EKF method. It was demonstrated that the proposed method is more time-saving and more accurate than the EKF method.
This paper is organized as follows: in Section II, the experiments and the data used in this research are explained in detail. Then in Section III, the model-driven prognosis method based on FDKF is addressed. The framework of the method is given, as well as the detailed explanation of FDKF. Four voltage degradation models are also chosen here. The result is obtained and analyzed in Section IV. Two case studies are applied to verify the method, and different models and training time are researched. The result of EKF is also compared with the FDKF method here. At the same time,the prediction horizon $(\mathrm{PH})$ is calculated and proved more accurate than the literature. Finally, the main conclusions are summarized in Section V.

To prevent confusion, rules of notation in this paper are as follows. The variables are taken in italic letters. The lowercase means that it is in the time domain, while uppercase means frequency domain. Meanwhile, a bold variable means vector and matrix, while the normal letter means that it is a scalar.

\section{EXPERIMENTS AND DATA}

\section{A. Experiments}

Two experiments were carried out in the Federation for Fuel Cell Research (FCLAB), where two PEMFC stacks degraded under different operating conditions [32]. The experiment platform is shown in figure 1. In the first experiment, a PEMFC stack with 5 fuel cells operated under a constant current of 70 $\mathrm{A}$, and it lasted for more than $1100 \mathrm{~h}$. It is called PEMFC1 in short. The main operating parameters of the experiment are shown in table I. The second experiment was carried out on another 5-cells stack, but under quasi-dynamic current, i.e., 70 A with 7 A high-frequency ripples, and it is called PEMFC2. The main operating parameters of PEMFC2 are shown as table II. The information of the stacks was recorded during the experiments, including the temperature, pressure, current, and voltage, etc.. There were characterization experiments about every week during both of the degradation experiments, including the polarization curve and electrochemical impedance spectroscope (EIS) measurement. The voltage has an obvious recovery after the characterization experiments, which enhances the nonlinear property of voltage. More details about the experiments can be found in the reference [32].

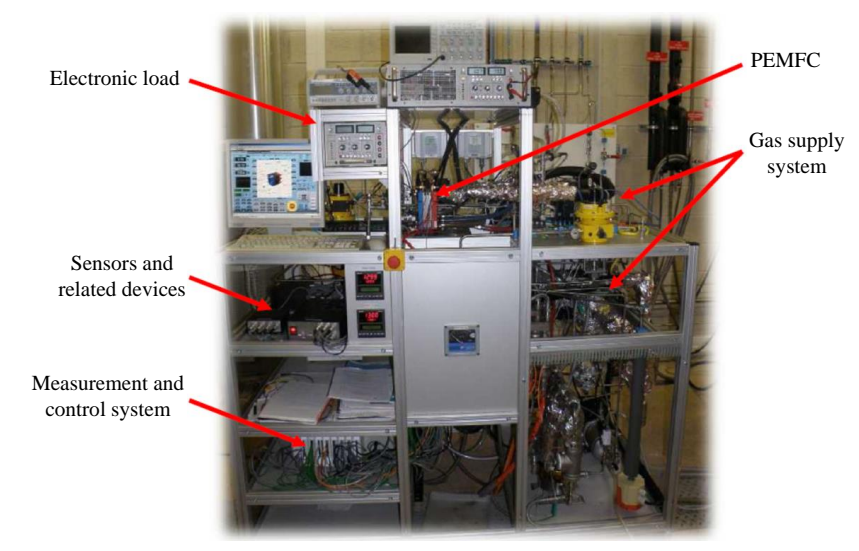

Fig. 1. PEMFC degradation experiment platform in FCLAB [32] 
TABLE I

EXPERIMENT PARAMETERS OF PEMFC1

\begin{tabular}{cc}
\hline Parameter & Value \\
\hline Number of cells & 5 \\
Active area & $100 \mathrm{~cm}^{2}$ \\
Stack rated current & $70 \mathrm{~A}$ \\
Temperature & $54{ }^{\circ} \mathrm{C}$ \\
Hydrogen pressure & $1.3 \mathrm{bar}$ \\
Relative humidity & $50 \%$ \\
\hline
\end{tabular}

TABLE II

EXPERIMENT PARAMETERS OF PEMFC2

\begin{tabular}{cc}
\hline Parameter & Value \\
\hline Number of cells & 5 \\
Active area & $100 \mathrm{~cm}^{2}$ \\
Stack rated current & $70 \mathrm{~A}$ with $7 \mathrm{~A}$ vibration \\
Temperature & $54^{\circ} \mathrm{C}$ \\
Hydrogen pressure & $1.3 \mathrm{bar}$ \\
Relative humidity & $51.8 \%$ \\
\hline
\end{tabular}

\section{B. Data processing}

The voltage data has to be processed before it is applied to the prognosis because there are random errors and violent fluctuations during the experiment. Gaussian kernel smooth method [33] is applied to process the voltage data in this research. It gives a weight to every data according to Gaussian distribution and then gets the weighted average as the new value. However, it should be noticed that the future data should not be used to smooth the data in prognosis, thus to prevent data leakage. Therefore, only the left half of the Gaussian distribution is applied, while the right half is set as 0 . For a time series data $d\left(t_{i}\right)$, where $i$ is the index of the data, $t_{i}$ is the corresponding time, the processed data $p\left(t_{i}\right)$ can be obtained as:

$$
p\left(t_{i}\right)=\frac{\sum_{t_{j}=t_{i}-\Delta t}^{t_{i}} K\left(t_{j}\right) \cdot d\left(t_{j}\right)}{\sum_{t_{j}=t_{i}-\Delta t}^{t_{i}} K\left(t_{j}\right)}
$$

Where $\Delta t$ is the smoothing interval, which is set $10 \mathrm{~h}$ in this case. The $K\left(t_{j}\right)$ is the Gaussian kernel function as:

$$
K\left(t_{j}\right)=\frac{1}{\sqrt{2 \pi}} e^{\frac{-\left(t_{j}-t_{i}\right)^{2}}{2 h^{2}}}
$$

Where the $h$ is the bandwidth, which is set as 500. The raw data and processed data of voltages are shown in figure 2(a) and 2(b) for PEMFC1 and PEMFC2, respectively. The corresponding operation currents of PEMFC1 and PEMFC2 are also shown in the figures. It can be seen that the processed data is smoother, but it can also represent the trend of original data. Afterward, only the processed data is used.

\section{Prognosis Method BASEd on FDKF}

In this part, the model-driven prognosis method based on FDKF is presented. First of all, the framework of the model-

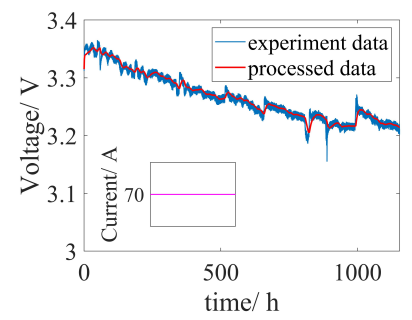

(a)

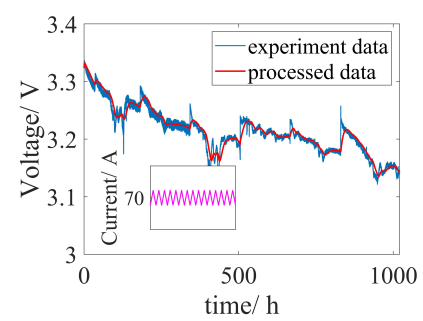

(b)

Fig. 2. Voltage raw data and processed data for (a) PEMFC1 and (b) PEMFC2

driven method is discussed in Section III-A. Then, the supposed voltage degradation models are given in Section III-B. Thirdly, the FDKF is explained in detail in Section III-C. Finally, the method to apply FDKF in the prognosis problem is addressed in Section III-D.

\section{A. Model-driven prognosis method based on FDKF}

Model-driven prognosis methods are based on models that describe the degradation phenomenon in PEMFC. The overall framework of the model-driven prognosis method based on FDKF is shown as figure 3. Firstly, the operation data of two experiments are used. During the experiment, the temperature, pressure, current, and voltage data are all recorded. Then the characterization of the data can decide which kinds of data will be used in the prognosis. In the PEMFC prognosis problem, stack voltage is usually easy to obtain and it can represent the degradation of PEMFC, so it is taken as an indicator of the state of health of PEMFC. Thirdly, as has been discussed in the introduction, model-driven methods rely on degradation models, so four empirical models are chosen in this research. Then the experimental data can be used to get the estimation of the state variables and output voltage by the proposed FDKF method. Furthermore, the degradation can be predicted by the FDKF method based on the state variables during the training period, thus the prognosis can be achieved.

\section{B. PEMFC voltage degradation models}

The degradation of PEMFC has been researched by experiments as well as theoretical analysis. However, as the degradation is a nonlinear and multi-physics process, it is difficult to get an exact model that perfectly corresponds to reality. In most research, it is given as a function of time with some undetermined parameters. Based on the Ref. [17] [34] [35], four different voltage degradation models are applied in this research. Namely, linear model, quadratic model, logarithmic 


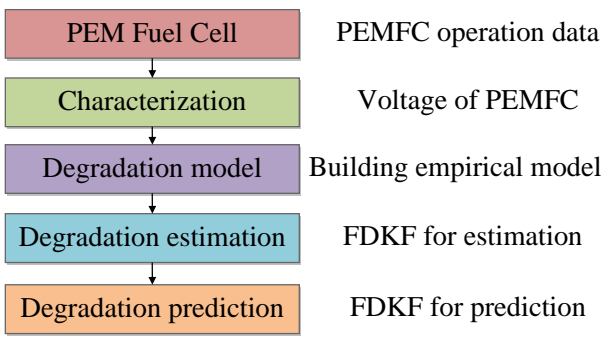

Fig. 3. Model-driven prognosis method based on FDKF for PEMFC

model and exponential model, which are shown as equations $3,4,5$ and 6 , respectively.

$$
\begin{gathered}
\text { Linear model }: x_{i+1}=x_{i}-\alpha \\
\text { Quadratic model }: x_{i+1}=x_{i}-\alpha-\beta \cdot i \\
\text { Logarithmic model }: x_{i+1}=x_{i}-\alpha-\beta \cdot \ln \left(\frac{i+1}{i}\right) \\
\text { Exponential model }: x_{i+1}=x_{i}-\alpha-\beta \cdot\left(e^{\gamma \cdot i}-e^{\gamma \cdot i-\gamma}\right)
\end{gathered}
$$

Where $x$ is the PEMFC voltage; $i$ is the time index; $\alpha, \beta, \gamma$ are undetermined parameters. The $\alpha$ is related to the voltage degradation rate under constant operating condition, and $\beta, \gamma$ are the parameters related to voltage degradation under dynamic operating conditions, such as load current change. The degradation of PEMFC is in a near-linear trend under constant load and constant operating condition [35]. When the load changes, the PEMFC will have some transient processes, such as gas diffusion and water accumulation. During the transient process, the degradation is usually accelerated due to the worse environment for the components such as a catalyst, and it is usually in a logarithmic or exponential modes [19].

The linear model is widely used to describe the voltage degradation under normal condition in a lot of references [17]. However, when the operating condition changes, this model is not enough to describe the degradation, therefore, other terms are introduced to amend it. So the quadratic term, logarithmic term, and exponential term are added in other models, respectively, so that to capture the behaviors under transient operating conditions [34]. Here the parameters in the proposed models are fitted by the least square method based on the training data.

\section{Frequency domain Kalman filter}

Originally, FDKF is widely used in AEC problem [36] [37]. In this part, we adapt the fuel cell prognosis problem into the form that can be solved by FDKF, which is shown as figure 4.

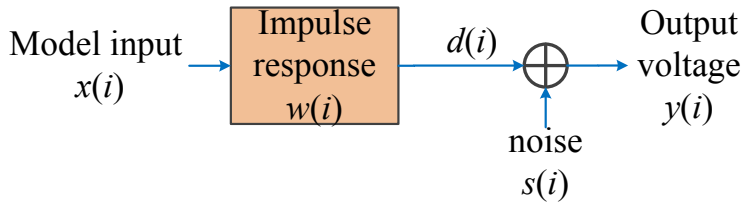

Fig. 4. Fuel cell prognosis problem in time domain
Here $i$ is the time index. $x(i)$ is the voltage signal from model. $w(i)$ is the impulse response path, which is unknown parameter decided by the fuel cell and the operating condition. The convolution of $x(i)$ and $w(i)$ forms $d(i) . s(i)$ is a white noise, and it forms the output signal $y(i)$ together with $d(i)$.

In this problem, the most important task is to find the path $w(i)$, so that to get rid of the noise and obtain $d(i)$. The relationship between $x(i), w(i)$ and $d(i)$ can be shown as:

$$
d(i)=x(i) * w(i)
$$

Here the $*$ is the convolution operator. So for $y(i)$, we have:

$$
\begin{aligned}
y(i) & =d(i)+s(i) \\
& =x(i) * w(i)+s(i)
\end{aligned}
$$

To solve this problem, we can change the equations from the time domain to the frequency domain, so Discrete Fourier Transfer (DFT) operation is applied to a series of data. To achieve DFT, a window of data at length $M$ is taken, and the window moves forward at a speed of $R$ data each step. In this research we take $M=256$ and $R=128$. For step $k$, take a vector to represent the latest $M$ data of $x(i)$ :

$\boldsymbol{x}(k)=[x((k-1) R+1), x((k-1) R+2), \ldots, x((k-1) R+M)]^{H}$

Where the superscript $H$ means the Hermitian transposition. So we get frequency domain input $\boldsymbol{X}(k)$ as:

$$
\boldsymbol{X}(k)=\operatorname{diag}\{\boldsymbol{F} \boldsymbol{x}(k)\}
$$

Where $\operatorname{diag}\{\}$ creates a new matrix that takes the vector as the main diagonal items of it. $\boldsymbol{F}$ is the Fourier matrix which can achieve the effect of Fourier transfer of a vector, thus changes the vector from the time domain to the frequency domain.

$$
\boldsymbol{F} \boldsymbol{x}(k)=[X(1, k), X(2, k), \ldots, X(M, k)]^{H}
$$

For $w(k)$, only the first $M-R$ order response is used, which is obtained from the last step, and we suppose that it can cover all the span of the impulse response. For the part where we ignore, add 0 to make it a $M$ data vector.

$$
\boldsymbol{w}(k)=[w(1, k), w(2, k), \ldots, w(M-R, k), 0, \ldots, 0]^{T} ;
$$

Also, in the frequency domain, we can get the complex frequency domain impulse response path as:

$$
\begin{aligned}
\boldsymbol{W}(k) & =\boldsymbol{F} \boldsymbol{w}(k) \\
& =[W(1, k), W(2, k), \ldots, W(M, k)]^{T}
\end{aligned}
$$

For $y(i)$ and $s(i)$, take the latest $R$ data as a vector, it can be represented as:

$$
\begin{aligned}
\boldsymbol{y}(k)= & {[y((k-2) R+M+1), y((k-2) R+M+2),} \\
& \ldots, y((k-1) R+M)]^{T} \\
\boldsymbol{s}(k)= & {[s((k-2) R+M+1), s((k-2) R+M+2),} \\
& \ldots, s((k-1) R+M)]^{T}
\end{aligned}
$$


Take $\boldsymbol{Q}^{H}=\left[\mathbf{0}_{R \times(M-R)} \boldsymbol{I}_{R \times R}\right]$, which is a $R$ row $M$ column matrix. It can take only the last $R$ items from a vector of length $M$. According to the of the convolution theory, the convolution of the time domain signal can be transferred to multiplication in the frequency domain, so we have:

$$
\boldsymbol{d}(k)=\boldsymbol{Q}^{H} \boldsymbol{F}^{-1} \boldsymbol{X}(k) \boldsymbol{W}(k)
$$

The equation 8 can be written as:

$$
\begin{aligned}
\boldsymbol{y}(k) & =\boldsymbol{d}(k)+\boldsymbol{s}(k) \\
& =\boldsymbol{Q}^{H} \boldsymbol{F}^{-1} \boldsymbol{X}(k) \boldsymbol{W}(k)+\boldsymbol{s}(k)
\end{aligned}
$$

Change it into the frequency domain and we can get:

$$
\boldsymbol{Y}(k)=\boldsymbol{F} \boldsymbol{Q} \boldsymbol{Q}^{H} \boldsymbol{F}^{-1} \boldsymbol{X}(k) \boldsymbol{W}(k)+\boldsymbol{F} \boldsymbol{Q} \boldsymbol{s}(k)
$$

Here the $Q$ is used to add 0 in the first $M-R$ data of a vector, to make a vector change from length $R$ to length $M$. Then, by taking:

$$
\begin{gathered}
\boldsymbol{C}(k)=\boldsymbol{F} \boldsymbol{Q} \boldsymbol{Q}^{H} \boldsymbol{F}^{-1} \boldsymbol{X}(k) \\
\boldsymbol{S}(k)=\boldsymbol{F} \boldsymbol{Q} \boldsymbol{s}(k)
\end{gathered}
$$

We can get:

$$
\boldsymbol{Y}(k)=\boldsymbol{C}(k) \boldsymbol{W}(k)+\boldsymbol{S}(k)
$$

We can see that this equation gives the relationship between the frequency domain input signal $\boldsymbol{X}(k)$, frequency domain impulse response $\boldsymbol{W}(k)$, and the frequency domain output signal $\boldsymbol{Y}(k)$.

By taking $\boldsymbol{W}(k)$ as state variable, $\boldsymbol{C}(k)$ as measurement matrix, $\boldsymbol{Y}(k)$ as output and $\boldsymbol{S}(k)$ as noise, supposing the frequency domain response $\boldsymbol{W}(k)$ only change slowly between two steps, we can get the system equations as:

$$
\left\{\begin{aligned}
\boldsymbol{W}(k+1) & =A \boldsymbol{W}(k)+\Delta \boldsymbol{W}(k) \\
\boldsymbol{Y}(k) & =\boldsymbol{C}(k) \boldsymbol{W}(k)+\boldsymbol{S}(k)
\end{aligned}\right.
$$

Where $A$ is a constant close to 1 . Take $\Delta \boldsymbol{W}(k)$ as white noise, then, in the frequency domain, the system can be represented as:

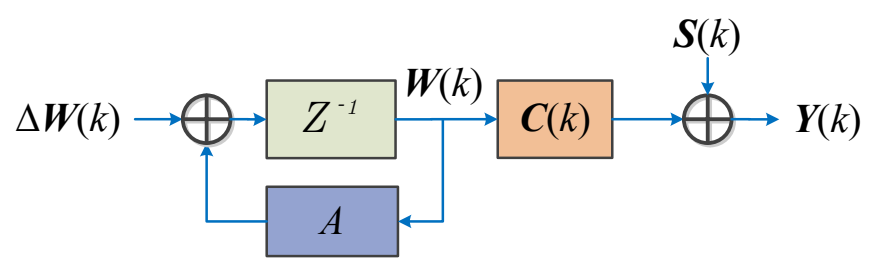

Fig. 5. System of fuel cell prognosis problem in frequency domain

For the problems like figure 5, we can use Kalman filter to obtain $\boldsymbol{W}(k)$. Suppose that $\boldsymbol{S}(k)$ and $\Delta \boldsymbol{W}(k)$ are uncorrelated Gaussian noise, with the covariance matrix as $\boldsymbol{\Phi}_{S S}$ and $\Phi_{\Delta \Delta}$, respectively. We can get the solution in Kalman filter form:

$$
\boldsymbol{W}^{+}(k)=A \boldsymbol{W}(k-1)
$$

$$
\begin{gathered}
\boldsymbol{P}^{+}(k)=A \boldsymbol{P}(k-1) A^{\prime}+\boldsymbol{\Phi}_{\Delta \Delta} \\
\boldsymbol{K}(k)=\boldsymbol{P}^{+}(k) \boldsymbol{C}^{H}(k)\left[\boldsymbol{C}(k) \boldsymbol{P}^{+}(k) \boldsymbol{C}^{H}(k)+\boldsymbol{\Phi}_{S S}\right]^{-1} \\
\boldsymbol{W}(k)=\boldsymbol{W}^{+}(k)+\boldsymbol{K}(k)[\boldsymbol{Y}(k)-\boldsymbol{C}(k) \boldsymbol{W}(k)] \\
\boldsymbol{P}(k)=(\boldsymbol{I}-\boldsymbol{K}(k) \boldsymbol{C}(k)) \boldsymbol{P}^{+}(k)
\end{gathered}
$$

Where the $\boldsymbol{P}(k)$ is the estimation error covariance, $\boldsymbol{W}^{+}(k)$ and $\boldsymbol{P}^{+}(k)$ means the step ahead estimation of $\boldsymbol{W}(k)$ and $\boldsymbol{P}(k) . \boldsymbol{K}(k)$ is the Kalman gain in the frequency domain. By this method, we can update $\boldsymbol{W}(k)$ step by step, then use it to predict the voltage output. The stability and convergence of the proposed FDKF method is given in the Appendix.

In this research, the parameters $A, \boldsymbol{\Phi}_{S S}$, and $\boldsymbol{\Phi}_{\Delta \Delta}$ are tuning parameters, and they are chosen according to the criterion that the estimated voltage is the closest to the experimental data. So $A=0.9999, \boldsymbol{\Phi}_{S S}=10^{-2} \boldsymbol{I}_{M \times M}$, and $\boldsymbol{\Phi}_{\Delta \Delta}=10^{-4} \boldsymbol{I}_{M \times M}$ are applied.

\section{Prognosis by FDKF}

In this part, we summarize the method to solve the prognosis problem with FDKF, and the diagram of the method is shown as figure 6 . The prognosis process can be divided into two parts, i.e., the learning period and the prediction period. In the learning period, by the models given in Section III-B, the $x(i)$ can be obtained. Group $x(i)$ in a block of $M$ data, and at each step the DFT of it can give the frequency domain input $\boldsymbol{X}(k)$. By frequency domain impulse response $\boldsymbol{W}(k)$, the estimated frequency domain output $\hat{\boldsymbol{Y}}(k)$ can be obtained. At the same time, the real output data $y(i)$ is also transferred into the frequency domain, which is $\boldsymbol{Y}(k)$. Then the frequency domain error $\boldsymbol{E}(k)$ can be obtained by the subtraction of real frequency domain output $\boldsymbol{Y}(k)$ and the estimated output $\hat{\boldsymbol{Y}}(i)$. It will be used to update $\boldsymbol{W}(k)$, according to the equations 23 to 27. Finally, the Inverse Discrete Fourier Transform (IDFT) can change the estimated frequency domain output $\hat{\boldsymbol{Y}}(k)$ into the time domain. By this method, the impulse response updates and the output voltage can be estimated.

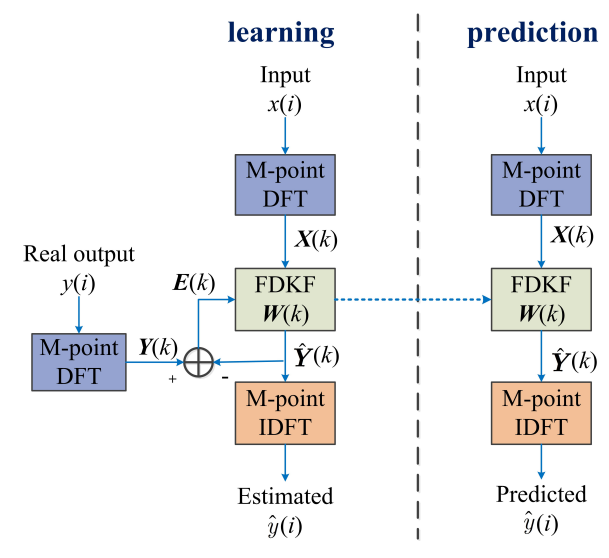

Fig. 6. PEMFC prognosis by FDKF

When the learning period is over, the process moves to the prediction period. During the prediction period, the $\boldsymbol{W}(k)$ of the learning period is used in the prediction. By the same process as the learning period, the $x(i)$ can be transferred to 
the frequency domain, and the predicted $\hat{\boldsymbol{Y}}(k)$ can be obtained by $\boldsymbol{X}(k)$ and $\boldsymbol{W}(k)$ according to equation 22 . At last, the predicted voltage $\hat{y}(i)$ can be calculated by IDFT, thus the prediction of voltage degradation is achieved.

\section{RESULT AND VALIDATION}

In this section, the prediction of PEMFC voltage is obtained according to the proposed method. Two case studies are carried out, where the experimental data of PEMFC1 is used in Section IV-A while PEMFC2 is used in Section IV-B. At the same time, the EKF method is compared with the proposed FDKF method in Section IV-C.

In each case study, four different models are studied respectively, and predictions are carried out under different training length so that to achieve both short-term and longterm prediction. The models are trained under $50 \%, 60 \%, 70 \%$, $80 \%, 90 \%$ of the total time respectively, and the rest part of the experimental data is used as verification. Mean Relative Error (MRE) and Root Mean Square Error (RMSE) are taken as the judgments of its performance, which can be calculated by equation 28 and 29 , respectively.

$$
\begin{gathered}
M R E=\frac{1}{N} \sum_{i=1}^{N}\left|\frac{\hat{V}(i)-V(i)}{V(i)}\right| \\
R M S E=\sqrt{\frac{\sum_{i=1}^{N}(\hat{V}(i)-V(i))^{2}}{N}}
\end{gathered}
$$

In the equations the $N$ is the number of data; $\hat{V}(i)$ is the predicted voltage; $V(i)$ is the real voltage output. The MRE is the mean relative errors, while RMSE is more sensitive to the big errors. By those two methods, the performance of a prediction can be measured.

\section{A. Case study 1: voltage degradation prediction for PEMFC1}

In this part, based on the PEMFC1 data, four voltage degradation models are researched under different training time. The predicted voltage and relative error are analyzed. They are given in the following order: linear model, quadratic model, logarithmic model, and exponential model.

1) Linear model for PEMFC1: The training and prediction voltage and corresponding relative error under linear model for PEMFC1 are shown as figure 7(a) and 7(b), respectively. It can be seen that the training result follows the experimental data well, and the predicted voltage is close to the real output voltage. As there are characterization experiments during the degradation experiment at some chosen points, the voltage sharply fluctuates and it is quite nonlinear. The predicted voltage is affected by the training length, and the result is more accurate with longer training time.

The relative error of the training period is smaller than $0.4 \%$, so it follows the training data well. For the prediction period, the error arises when the experimental voltage abruptly changes, but the prediction error is always smaller than $1.8 \%$, and the averaged RMSE is $0.0327 \mathrm{~V}$. The experiment voltage suddenly rises at about 1000 hours, which is not recognized by the long-term prediction, thus the error is relatively big.
However, the predicted voltage under 90\% training time corresponds well with the experiment. In summary, as some nonlinear behaviors of PEMFC in the future are not considered, the long-term prediction is less accurate than short-term prediction.

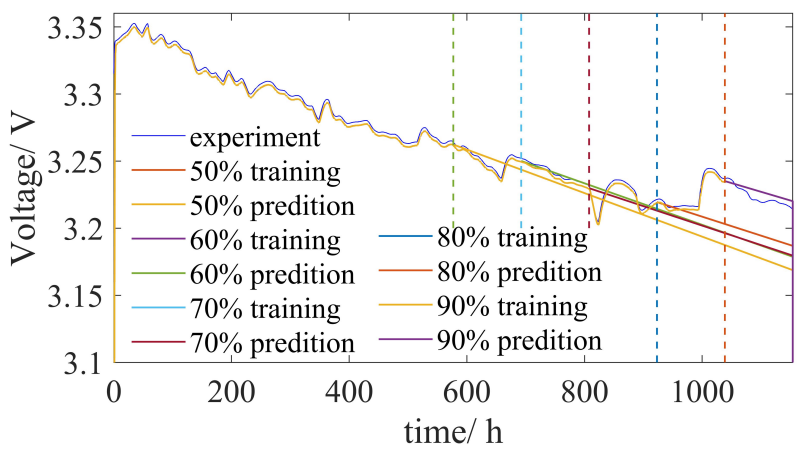

(a)

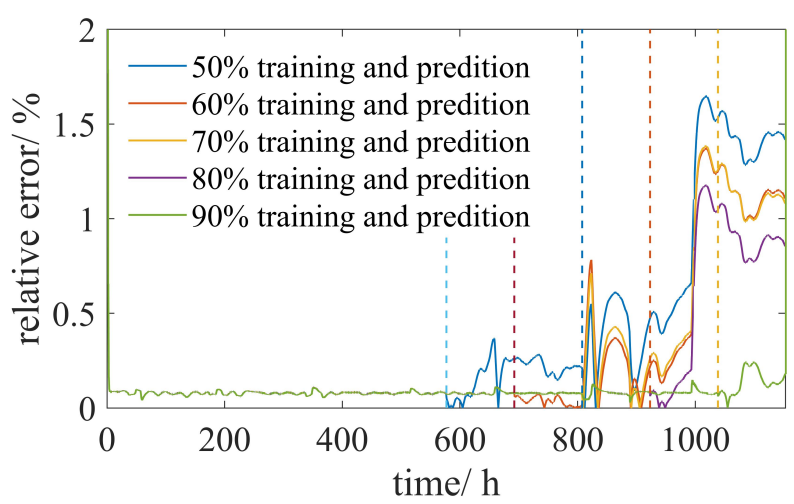

(b)

Fig. 7. (a) The voltage prediction result based on FDKF under linear model and (b) the relative error under linear model for PEMFC1

2) Quadratic model for PEMFC1: The quadratic model is the combination of a linear term and a quadratic term. Different training time is researched in this case, and the predicted voltage and relative error are shown as figure 8(a) and 8(b), respectively. As shown in figure 8(a), the predicted voltage can follow the actual voltage degradation trend for PEMFC1. As shown in figure $8(\mathrm{~b})$, the relative error of this model is always smaller than $1.4 \%$, while the maximum error of the linear model is $1.8 \%$. The reason for the high accuracy of the quadratic model is that the quadratic term effectively considers the voltage recovery phenomenon of PEMFC1 caused by the characteristic experiment.

3) Logarithmic model for PEMFCl: The logarithmic model is the combination of a linear term and a logarithmic term. The training and prediction result and corresponding relative error under logarithmic model for PEMFC1 are shown as figure 9(a) and 9(b), respectively. It can be seen that the result of the logarithmic model is quite similar to the linear model, and it is more accurate than the linear model, which is shown by the average MRE in table III. The reason for the high accuracy of the logarithmic model is that the logarithmic term effectively considers the voltage recovery phenomenon of 


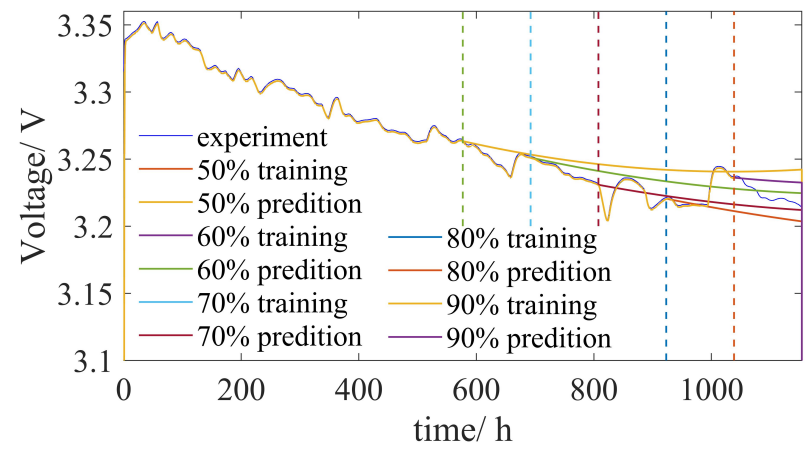

(a)

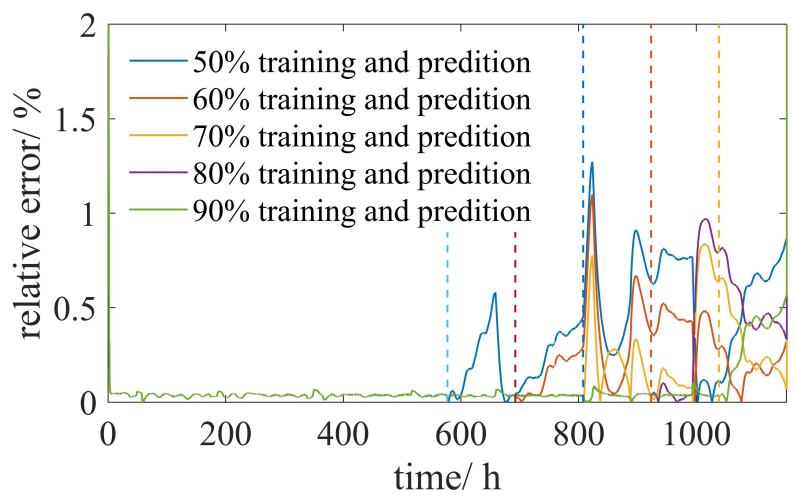

(b)

Fig. 8. (a) The voltage prediction result based on FDKF under quadratic model and (b) the relative error under quadratic model for PEMFC1

PEMFC1 caused by the characteristic experiment. It is notable that the result is quite accurate before the sudden jump of voltage at $1000 \mathrm{~h}$, where the relative error is less than $1 \%$. Therefore, for some works that declares the end-of-life (EOL) of the stack at $800 \mathrm{~h} \mathrm{[15],} \mathrm{the} \mathrm{prediction} \mathrm{can} \mathrm{be} \mathrm{more} \mathrm{accurate}$ because the voltage degradation after EOL isn't concerned.

4) Exponential model for PEMFC1: For the exponential model, the voltage result and corresponding relative error for PEMFC1 are shown as figure $10(\mathrm{a})$ and $10(\mathrm{~b})$, respectively. It can be seen that the result under $70 \%$ and $80 \%$ training time is accurate compared with experiment voltage, while the relative error under other training time is huge. The predicted voltage goes up dramatically because of the existence of an exponential term, which is far from reality. This proves that the exponential model is not robust for different training time, thus it is not proper to be used in the method.

5) Comparison of different models: To compare those four models, the MRE and RMSE of predictions under different models and different training time are listed in table III and table IV, respectively. It can be seen that the average MRE of the quadratic model is the smallest, which is $0.4419 \%$, and the average MRE of the linear model and logarithmic model are very close to that of the quadratic model, which are $0.6131 \%$ and $0.5999 \%$, respectively. All those three models can give an acceptable result. They are also applied to PEMFC2 in the next section, to verify if they are effective under the quasidynamic current condition. The RMSE of the predictions give

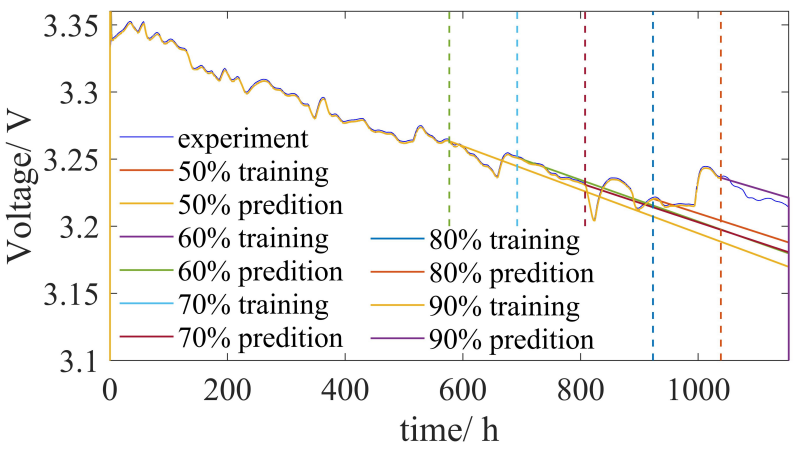

(a)

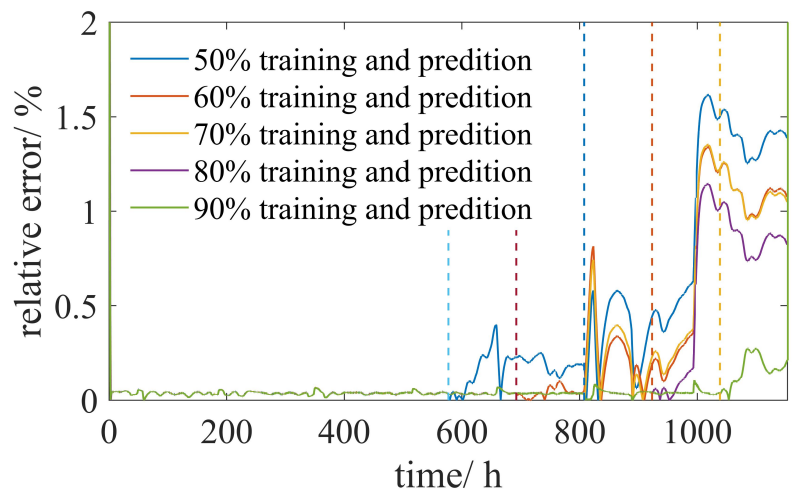

(b)

Fig. 9. (a) The voltage prediction result based on FDKF under logarithmic model and (b) the relative error under logarithmic model for PEMFC1

the same conclusions as MRE. As we can see, the RMSE of the exponential model is much higher than the linear model, quadratic model, and logarithmic model, thus the exponential model is unacceptable.

TABLE III

MRE (\%) OF PREDICTION UNDER DIFFERENT MODEL AND DIFFERENT TRAINING TIME FOR PEMFC1

\begin{tabular}{lcccccc}
\hline model & $50 \%$ & $60 \%$ & $70 \%$ & $80 \%$ & $90 \%$ & average \\
\hline linear & 0.6825 & 0.5302 & 1.0096 & 0.6698 & 0.1736 & 0.6131 \\
quadratic & 0.5449 & 0.3423 & 0.5276 & 0.4087 & 0.3861 & 0.4419 \\
logarithmic & 0.6593 & 0.5142 & 0.9808 & 0.6438 & 0.2014 & 0.5999 \\
exponential & 4.6888 & 0.5811 & 0.3844 & 0.3523 & 1.7269 & 1.5466 \\
\hline
\end{tabular}

TABLE IV

RMSE (V) OF PREDICTION UNDER DIFFERENT MODEL AND DIFFERENT TRAINING TIME FOR PEMFC1

$\begin{array}{lllllll}\text { model } & 50 \% & 60 \% & 70 \% & 80 \% & 90 \% & \text { average }\end{array}$

$\begin{array}{llllllllllll}\text { linear } & 0.0477 & 0.0357 & 0.0466 & 0.0273 & 0.0060 & 0.0327\end{array}$

$\begin{array}{lllllllll}\text { quadratic } & 0.0306 & 0.0115 & 0.0202 & 0.0146 & 0.0135 & 0.0181\end{array}$

$\begin{array}{llllllll}\text { logarithmic } & 0.0467 & 0.0347 & 0.0456 & 0.0263 & 0.0070 & 0.0320\end{array}$ exponential $0.4127 \quad 0.03210 .01240 .01190 .0662 \quad 0.1071$ 


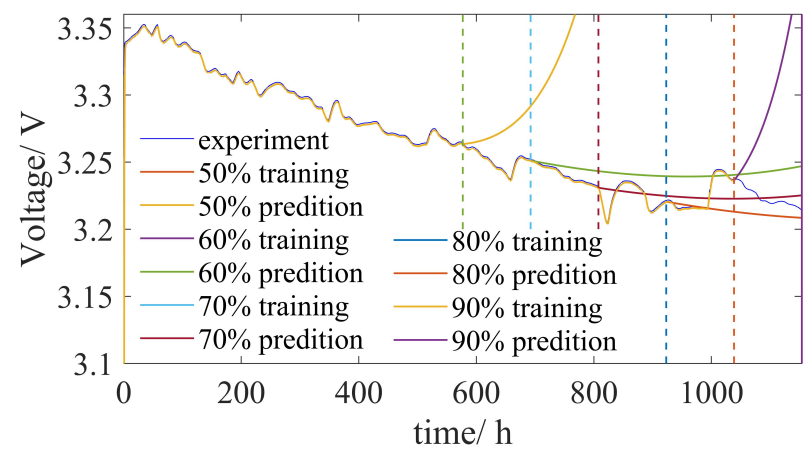

(a)

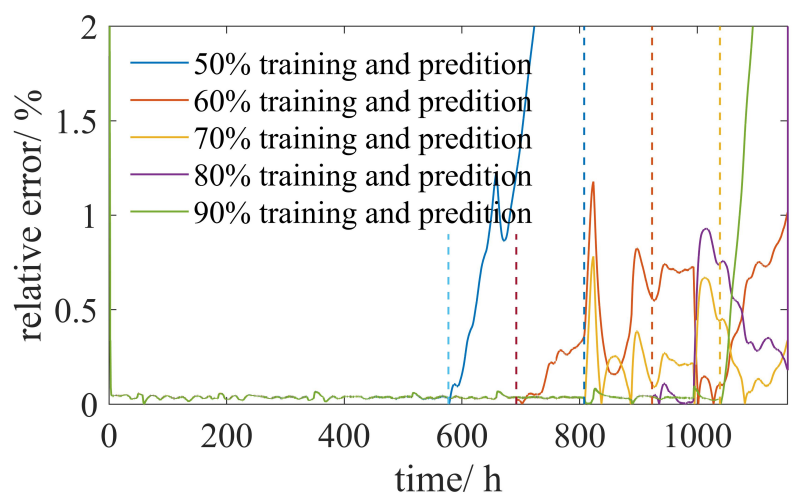

(b)

Fig. 10. (a) The voltage prediction result based on FDKF under exponential model and (b) the relative error under exponential model for PEMFC1

\section{B. Case study 2: voltage degradation prediction for PEMFC2}

The experiment PEMFC2 is carried out under a quasidynamic current of $70 \mathrm{~A}$ with $7 \mathrm{~A}$ high-frequency ripples, whose operation conditions and voltage output can be found in Section II. The degradation pattern of PEMFC2 is different from PEMFC1, as PEMFC1 is obtained under constant current. To verify the FDKF prognosis method under the quasi-dynamic current case, it is also applied to PEMFC2. The FDKF method is also trained under 50\%, 60\%, 70\%, $80 \%, 90 \%$ of the total time respectively, and the rest part of the experimental data is used as verification. In this section, the quadratic model, linear model, and logarithmic model are calculated, as the exponential model has already been proved unsuitable to be used in this method by the research above.

1) Quadratic model for PEMFC2: As the quadratic model has the smallest relative error in the PEMFC1 case, it is applied to PEMFC2 firstly. The training and prediction result and corresponding relative error under quadratic model for PEMFC2 are shown as figure 11(a) and 11(b), respectively. The result is very different under different training time. The model results under $60 \%$ and $70 \%$ training time corresponds badly with the voltage of PEMFC2, which could be caused by the nonlinear feature of PEMFC2. Compared with the result of PEMFC1 based on the quadratic model, the quadratic term cannot accurately model the voltage recovery phenomenon of PEMFC2. Therefore, this model is unsuitable to be used in this case, especially for long-term prediction. As the result of the linear model and logarithmic model are acceptable in PEMFC1, both of them are calculated for PEMFC2 by the proposed FDKF method.

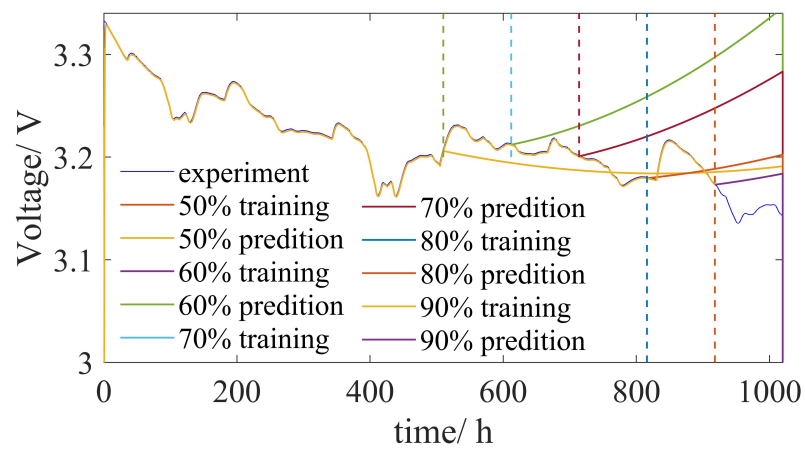

(a)

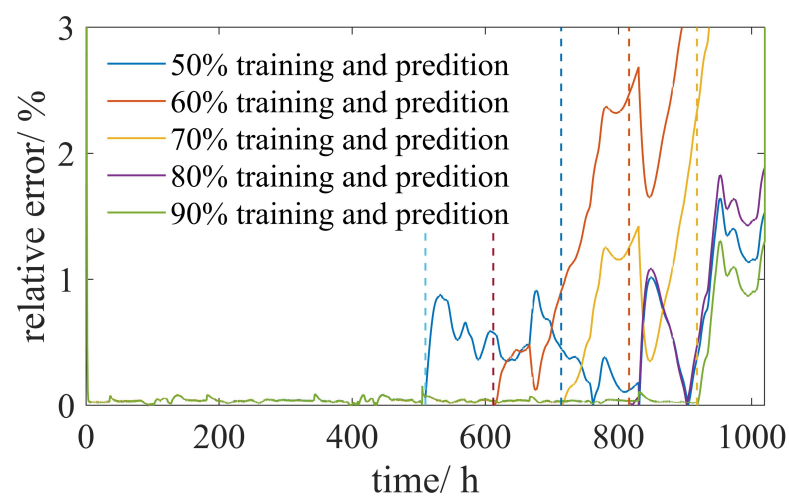

(b)

Fig. 11. (a) The voltage prediction result based on FDKF under quadratic model and (b) the relative error under quadratic model for PEMFC2

2) Linear model for PEMFC2: The voltage result and corresponding relative error under linear model for PEMFC2 is shown as figure 12(a) and 12(b), respectively. It can be seen that although the relative error arises when the voltage fluctuates, it is always smaller than $2.5 \%$, thus the result of the linear model is more accurate than the quadratic model. As can be seen in table $\mathrm{V}$, the average MRE of the linear model is $0.5300 \%$, which is much smaller than the quadratic model. The MRE of PEMFC2 is also smaller than the result of the linear model for PEMFC1. Thus, the linear model can be used in the FDKF method for both cases.

3) Logarithmic model for PEMFC2: For the logarithmic model, the training and prediction result and corresponding relative error for PEMFC2 are presented in figure 13(a) and 13(b), respectively. Again, the result is similar to that of the linear model. The predicted voltage is close to the actual degraded voltage, and the maximum relative error is less than $2.5 \%$. The average MRE of this model is $0.5291 \%$, which is slightly smaller than $0.5300 \%$ of the linear model, and it is also smaller than the MRE of the logarithmic model for PEMFC1. Compared with the result of PEMFC1 based on the logarithmic model, the logarithmic term can also accurately model the voltage recovery phenomenon of PEMFC2. Recall that the logarithmic model is also a little more accurate than the linear 


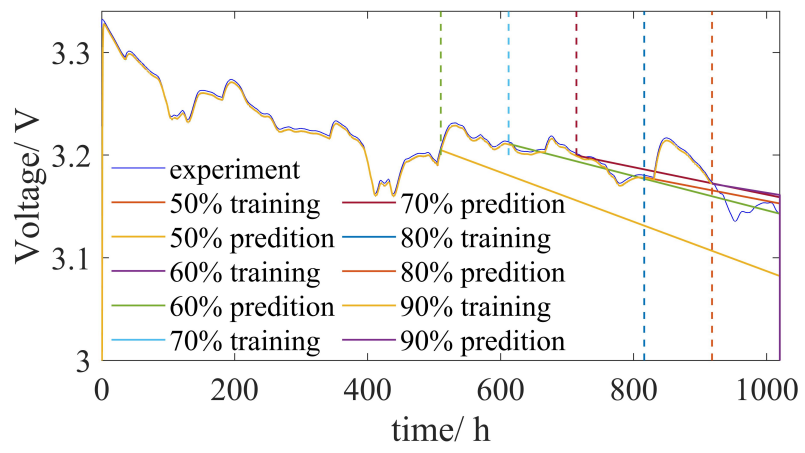

(a)

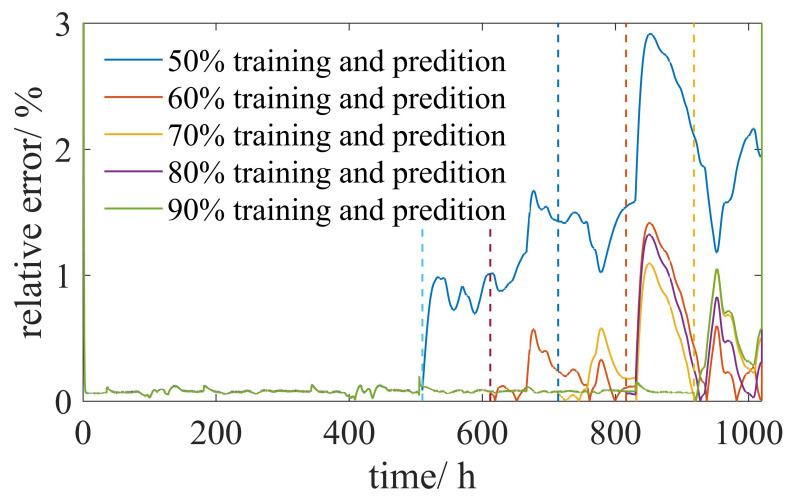

(b)

Fig. 12. (a) The voltage prediction result based on FDKF under linear model and (b) the relative error under linear model for PEMFC2

model for PEMFC1, we can conclude that the logarithmic model is the most suitable model when considering both PEMFC1 and PEMFC2. This agrees with the conclusion of the particle filter method [15].

The MRE and RMSE of different models under different training time for PEMFC2 are shown in table V and VI, respectively. It can be seen that although the MRE of the quadratic model is smaller than the linear model and logarithmic model under 50\% training time, the error is high with longer training time. Therefore, the quadratic model is unsuitable for this case. The errors of the linear and logarithmic model are both small enough under all training time, which proves that they can achieve both short-term and long-term prediction, thus they are robust for both two cases. The RMSE of the predictions shows the same tendency as MRE, i.e., the RMSE of linear and logarithmic models are much smaller than the quadratic model, thus both linear and logarithmic model are accurate. Both MRE and RMSE of the linear model and logarithmic models are very close, thus both MRE and RMSE can be used to evaluate the performance of prediction.

\section{Comparison with extended Kalman filter}

Kalman filter can also be applied to degradation prediction in the time domain. However, a normal Kalman filter cannot solve nonlinear problems. As an adaptation of the Kalman filter, Extended Kalman Filter (EKF) takes the Jacobi matrix

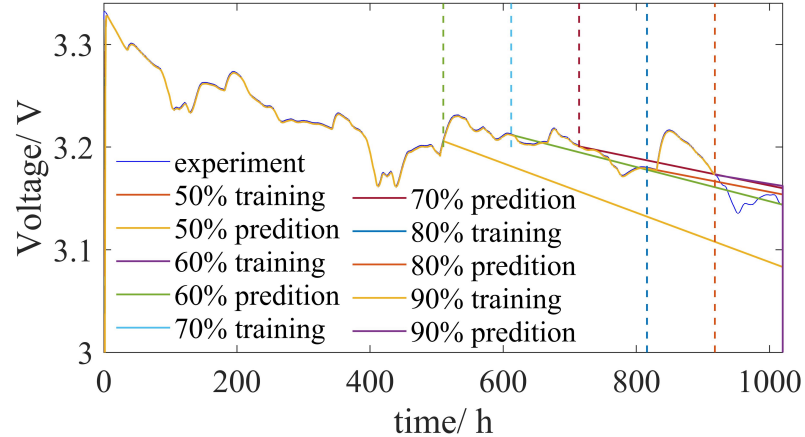

(a)

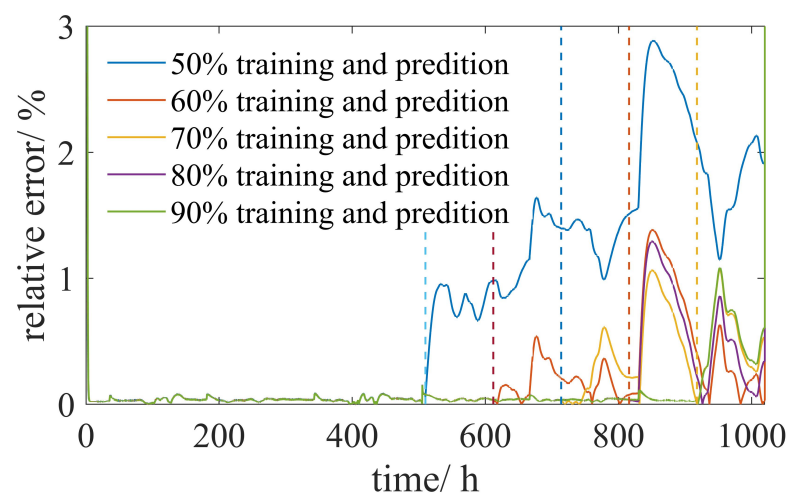

(b)

Fig. 13. (a) The voltage prediction result based on FDKF under logarithmic model and (b) the relative error under logarithmic model for PEMFC2

TABLE V

MRE (\%) OF PREDICTION UNDER DIFFERENT MODEL AND DIFFERENT TRAINING TIME FOR PEMFC2

\begin{tabular}{lcccccc}
\hline model & $50 \%$ & $60 \%$ & $70 \%$ & $80 \%$ & $90 \%$ & average \\
\hline quadratic & 0.7013 & 2.4230 & 1.7366 & 0.9723 & 0.7074 & 1.3081 \\
linear & 0.8867 & 0.3784 & 0.4506 & 0.5611 & 0.3729 & 0.5300 \\
logarithmic & 0.8545 & 0.3664 & 0.4595 & 0.5610 & 0.4041 & 0.5291 \\
\hline
\end{tabular}

TABLE VI

RMSE (V) OF PREDICTION UNDER DIFFERENT MODEL AND DIFFERENT TRAINING TIME FOR PEMFC2

\begin{tabular}{lcccccc}
\hline model & $50 \%$ & $60 \%$ & $70 \%$ & $80 \%$ & $90 \%$ & average \\
\hline quadratic & 0.0653 & 0.1668 & 0.1110 & 0.0442 & 0.0237 & 0.0822 \\
linear & 0.0335 & 0.0086 & 0.0185 & 0.0131 & 0.0138 & 0.0175 \\
logarithmic & 0.0325 & 0.0090 & 0.0195 & 0.0139 & 0.0147 & 0.0179 \\
\hline
\end{tabular}


of the nonlinear equation, so that it can be applied to a slightly nonlinear problem. The EKF method has been applied to the fuel cell prognosis and proved advanced [12] [38] [39], thus it is applied to the PEMFC prognosis problem here, and it is carried out with three models for both PEMFC1 and PEMFC2 experiments. The computation time as well as the relative error of prediction are obtained and compared with the FDKF method.

The total number of data used in this research is 1120000 and 1040000 for PEMFC1 and PEMFC2, respectively. Therefore, the sampling frequency is $0.278 \mathrm{~Hz}$. For both FDKF method and EKF method, the calculations are all carried out on under same test conditions, i.e., a personal computer with processor Intel(R)Xeon(R)W-2123CPU@ 3.60GHz; memory RAM 32GB; operation system Window 10 education and Matlab R2018a. For the linear model, quadratic model, and logarithmic model, the voltage degradation is predicted for both PEMFC1 and PEMFC2 by FDKF and EKF method, respectively. The computation time is shown as table VII. It can be seen that the FDKF method uses much less time than EKF for both experiments under all three models. The FDKF method can give results in several seconds, while the EKF method needs thousands of seconds. As has been addressed, the FDKF method is more efficient because it can process the data in groups, and it moves forward with $R$ data at each step, which is the advantage of FDKF.

TABLE VII

COMPUTATION TIME (S) UNDER DIFFERENT DEGRADATION MODELS FOR EKF AND FDKF

\begin{tabular}{lcccc}
\hline Method & \multicolumn{4}{c}{ linear quadratic logarithmic average } \\
\hline FDKF for PEMFC1 & 5.269 & 4.879 & 13.60 & 7.915 \\
EKF for PEMFC1 & 1506 & 2456 & 2452 & 2138 \\
FDKF for PEMFC2 & 4.319 & 4.422 & 11.20 & 6.647 \\
EKF for PEMFC2 & 1507 & 1932 & 1895 & 1628 \\
\hline
\end{tabular}

Compared with the EKF method, the FDKF method also has better performance in terms of accuracy. The MRE and RMSE under $50 \%, 60 \%, 70 \%, 80 \%$, and $90 \%$ training time are averaged to represent the performance of the method, and they are shown in table VIII and IX, respectively. As we can see, the relative error of the EKF method under the quadratic model is very big, while that of the FDKF method is acceptable. Thus FDKF method can be used under more models. Furthermore, the error of the FDKF method is smaller than the EKF method under all three models, and the RMSE can give the same conclusion, which proves that the proposed method is advantageous. This is due to the different characteristics of FDKF and EKF method. The FDKF method deals with the data in the frequency domain, and it finds the basic structure of the data in the frequency domain so that to find the invariance of the data. For EKF, it uses the Taylor's expansion to replace the nonlinear function, then only the first-order term is reserved and the terms of higher order are neglected. In this way, a linear system can be obtained and the Kalman filter can be applied to the linearized system. The performance of EKF is worse than FDKF in our case because the real system is highly nonlinear, thus the linearization process of the EKF method will introduce more error because of the neglected high-order terms. Therefore, the prediction error of the EKF method is bigger.

TABLE VIII

AVERAGED MRE (\%) UNDER DIFFERENT DEGRADATION MODELS FOR EKF AND FDKF

\begin{tabular}{lcccc}
\hline Method & \multicolumn{3}{c}{ linear } & quadratic logarithmic average \\
\hline FDKF for PEMFC1 & 0.6131 & 0.4419 & 0.5999 & 0.5516 \\
EKF for PEMFC1 & 0.7718 & 4.2740 & 0.7717 & 1.9392 \\
FDKF for PEMFC2 & 0.5300 & 1.3081 & 0.5291 & 0.7891 \\
EKF for PEMFC2 & 1.0775 & 6.1435 & 1.0755 & 2.7655 \\
\hline
\end{tabular}

TABLE IX

AVERAGED RMSE (V) UNDER DIFFERENT DEGRADATION MODELS FOR EKF AND FDKF

\begin{tabular}{lcccc}
\hline Method & linear & quadratic logarithmic average \\
\hline FDKF for PEMFC1 & 0.0327 & 0.0181 & 0.0320 & 0.0276 \\
EKF for PEMFC1 & 0.0345 & 0.1637 & 0.0345 & 0.0776 \\
FDKF for PEMFC2 & 0.0175 & 0.0822 & 0.0179 & 0.0392 \\
EKF for PEMFC2 & 0.0412 & 0.2344 & 0.0412 & 0.1056 \\
\hline
\end{tabular}

\section{Prediction horizon}

According to the references [40], the Prediction Horizon (PH) is an important index for prognosis, thus the $\mathrm{PH}$ is calculated and compared with the references here. The $\mathrm{PH}$ is a measurement of the accuracy of the Remaining Useful Life (RUL). At different prediction time, the real RUL and predicted RUL can be plotted to see the performance of the prediction. Usually, an acceptable error is set, which is a certain percent of the End of Life (EOL). After a certain prediction time, the predicted RUL will all located within the acceptable error region, thus we can indicate how long ago the acceptable prediction can be obtained and ensured. In this research, the error bound $\alpha$ is set as $10 \%$ according to most researches. The EOL can be declared when the performance of the PEMFC is smaller than a critical line. In this research the critical line is set as $95 \%$ of the initial voltage, thus the EOL is 936 hours. According to the proposed method, the RUL prediction and $\mathrm{PH}$ can be given as figure 14. The predicted RUL is within the acceptable region after 529 hours, thus the $\mathrm{PH}$ can be obtained as 407 hours.

In the reference [41], the particle filter was applied, and the voltage recovery phenomenon was considered. In their research, the $\mathrm{PH}$ without consideration of recovery phenomenon is 310 hours, and the $\mathrm{PH}$ by the model with consideration of recovery phenomenon is 380 hours. Therefore, the $\mathrm{PH}$ of the proposed method is longer, which means that the proposed method can give accurate prediction earlier, thus it is more accurate. 


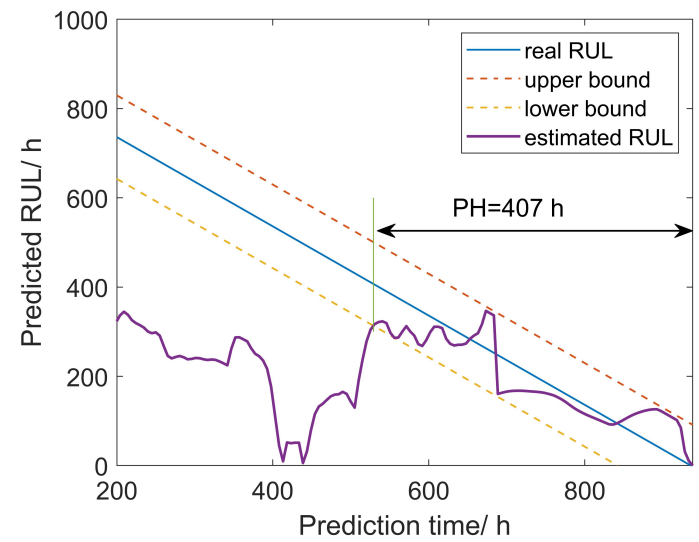

Fig. 14. The prediction horizon for PEMFC2 by logarithmic model

\section{CONCLusions}

In this paper, we proposed a novel model-driven method for the PEMFC prognosis problem, which is based on FDKF and voltage degradation model. The advantage of this method is that it can process data in groups, thus it is more efficient. The proposed method is verified by two case studies, and the voltage degradation is predicted both in short-term and long-term under 4 degradation models. The result of the FDKF method is also compared with EKF and literature. The conclusions are given as follows:

(1) With the proposed FDKF method, the quadratic model is only effective for PEMFC1, while both the linear and logarithmic models give acceptable results for both PEMFC1 and PEMFC2.

(2) In our research, the relative error of voltage degradation prediction is always smaller than $1.8 \%$ for PEMFC1, and it is smaller than $3 \%$ for PEMFC2. It proves that the proposed method is accurate and robust for both constant and quasidynamic current cases.

(3) The performance of the proposed FDKF method outweighs the EKF method both in terms of efficiency and accuracy. The proposed method can give results in several seconds, which is much more efficient than the EKF method. More importantly, the result of the FDKF method is also more accurate than the EKF method. The PH is also calculated and compared with other research, which proves that the proposed method is more accurate.

This method can also be applied to other kinds of fuel cells or some other fields where there are similarities between the problems. Combining the proposed FDKF method with energy management strategies to improve the durability and economy of fuel cells will be considered in future research work.

\section{APPENDIX}

\section{PROOF OF STABILITY AND CONVERGENCE OF FDKF METHOD}

The FDKF can be considered as a variable step-size frequency domain adaptive filter [36] [42], thus the solution can also be given as:

$$
\boldsymbol{W}(k+1)=A\left[\boldsymbol{W}(k)+\boldsymbol{Q} \boldsymbol{\mu}(k) \boldsymbol{\Lambda}^{-1}(k) \boldsymbol{C}^{H}(k) \boldsymbol{E}(k)\right]
$$

Where

$$
\begin{gathered}
\boldsymbol{E}(k)=\boldsymbol{Y}(k)-\boldsymbol{C}(k) \boldsymbol{W}(k) \\
\boldsymbol{\Lambda}(k)=\boldsymbol{C}^{H}(k) \boldsymbol{C}(k) \\
\boldsymbol{\mu}(k)=\boldsymbol{C}(k) \boldsymbol{P}(k) \boldsymbol{C}^{H}(k)\left[\boldsymbol{C}(k) \boldsymbol{P}(k) \boldsymbol{C}^{H}(k)+\boldsymbol{\Phi}_{S S}\right]^{-1} \\
=\operatorname{diag}\left\{\left[\mu_{0}(k), \mu_{1}(k), \ldots, \mu_{M}(k)\right]^{T}\right\}
\end{gathered}
$$

Where the $\boldsymbol{\mu}(k)$ is called the variable step-size matrix. Take equation 24 and 25 into equation 27, we can get:

$$
\boldsymbol{P}(k+1)=A^{2}\left[\boldsymbol{I}-\frac{1}{2} \boldsymbol{\mu}(k)\right] \boldsymbol{P}(k)+\boldsymbol{\Phi}_{\Delta \Delta}(k)
$$

As all the matrices are diagonal, every diagonal item in $\boldsymbol{P}(k)$ can be given as:

$$
\begin{aligned}
P_{i}(k+1)= & A^{2}\left[1-\frac{1}{2} \frac{P_{i}(k)}{P_{i}(k)+\Phi_{S S, i}(k) /\left|C_{i}(k)\right|^{2}}\right] \times P_{i}(k) \\
& +\Phi_{\Delta \Delta, i}(k)
\end{aligned}
$$

It
$A^{2}\left[1-\frac{1}{2} \frac{P_{i}(k)}{P_{i}(k)+\Phi_{S S, i}(k) /\left|C_{i}(k)\right|^{2}}\right]<1$ seen
that the coefficient thus the $P_{i}(k)$ can achieve an equilibrium after iterations. To find the steady state $P_{i}(\infty)$, we suppose that the far-end signal and noise signal are stationary, so that the $\Phi_{S S, i}(k) /\left|C_{i}(k)\right|^{2}$ can be replaced as $\eta_{i}$. And the $\Phi_{\Delta \Delta, i}(k)$ can be replaced by $\left(1-A^{2}\right) \Phi_{W W, i}(k)$. Therefore, the steady state is:

$P_{i}(\infty)=A^{2}\left(1-\frac{1}{2} \frac{P_{i}(\infty)}{P_{i}(\infty)+\eta_{i}}\right) P_{i}(\infty)+\left(1-A^{2}\right) \Phi_{W W, i}$

Then this equation can be solved and the positive solution is:

$$
\begin{aligned}
P_{i}(\infty)= & \frac{1-A^{2}}{2-A^{2}} . \\
& \left(\Phi_{W W, i}-\eta_{i}+\sqrt{\Phi_{W W, i}^{2}+\eta_{i}^{2}+\frac{2 \eta_{i} \Phi_{W W, i}}{1-A^{2}}}\right)
\end{aligned}
$$

The solution can be normalized by dividing both sides with $\Phi_{W W, i}$, thus the normalized prediction distance $\bar{P}_{i}(\infty)=P_{i}(\infty) / \Phi_{W W, i}$, and take $\delta_{i}=\Phi_{W W, i} / \eta_{i}$, and the solution can be given as:

$$
\bar{P}_{i}(\infty)=\frac{1-A^{2}}{2-A^{2}}\left(1-\frac{1}{\delta_{i}}+\sqrt{1+\frac{1}{\delta_{i}^{2}}+\frac{2}{\left(1-A^{2}\right) \delta_{i}}}\right)
$$

Take the solution into equation 33 , the steady state step size can be given as:

$$
\mu_{i}(\infty)=\frac{\delta_{i} \bar{P}_{i}(\infty)}{\delta_{i} \bar{P}_{i}(\infty)+1}
$$


Therefore for all $0<A \leq 1$, the FDKF is stable and can converge to a finite constant, and the converge rate can be decided by $A$ and $\delta_{i}$.

\section{ACKNOWLEDGMENT}

We gratefully acknowledge the support of this work by China Scholarship Council (CSC).

\section{REFERENCES}

[1] A. S. Abdelrahman, Y. Attia, K. Woronowicz, and M. Z. Youssef, "Hybrid fuel cell/battery rail car: A feasibility study," IEEE Transactions on Transportation Electrification, vol. 2, no. 4, pp. 493-503, 2016.

[2] T. Jahnke, G. Futter, A. Latz, T. Malkow, G. Papakonstantinou, G. Tsotridis, P. Schott, M. Gérard, M. Quinaud, M. Quiroga, A. A. Franco, K. Malek, F. Calle-Vallejo, R. Ferreira de Morais, T. Kerber, P. Sautet, D. Loffreda, S. Strahl, M. Serra, P. Polverino, C. Pianese, M. Mayur, W. G. Bessler, and C. Kompis, "Performance and degradation of proton exchange membrane fuel cells: State of the art in modeling from atomistic to system scale," Journal of Power Sources, vol. 304, pp. 207-233, 2016

[3] A. Chapman, K. Itaoka, H. Farabi-Asl, Y. Fujii, and M. Nakahara, "Societal penetration of hydrogen into the future energy system: Impacts of policy, technology and carbon targets," International Journal of Hydrogen Energy, vol. 45, no. 7, 2020.

[4] H. Homayouni, J. DeVaal, F. Golnaraghi, and J. Wang, "Voltage Reduction Technique for Use With Electrochemical Impedance Spectroscopy in High-Voltage Fuel Cell and Battery Systems," IEEE Transactions on Transportation Electrification, vol. 4, no. 2, pp. 418-431, 2018.

[5] P. Mungporn, P. Thounthong, B. Yodwong, C. Ekkaravarodome, A. Bilsalam, S. Pierfederici, D. Guilbert, B. Nahid-Mobarakeh, N. Bizon, and Z. Shah, "Modeling and Control of Multiphase Interleaved Fuel-Cell Boost Converter based on Hamiltonian Control Theory for Transportation Applications," IEEE Transactions on Transportation Electrification, 2020.

[6] J. Liu, Q. Li, Y. Han, G. Zhang, X. Meng, J. Yu, and W. Chen, "PEMFC residual life prediction using sparse autoencoder-based deep neural network," IEEE Transactions on Transportation Electrification., vol. 5, no. 4, pp. 1279-1293, 2019.

[7] B. Tanc, H. T. Arat, E. Baltacioglu, and K. Aydin, "Overview of the next quarter century vision of hydrogen fuel cell electric vehicles," International Journal of Hydrogen Energy, vol. 44, no. 20, pp. 10120 $10128,2019$.

[8] N. Sulaiman, M. A. Hannan, A. Mohamed, E. H. Majlan, and W. R. Wan Daud, "A review on energy management system for fuel cell hybrid electric vehicle: Issues and challenges," Renewable and Sustainable Energy Reviews, vol. 52, pp. 802-814, 2015.

[9] T. Sutharssan, D. Montalvao, Y. K. Chen, W.-C. Wang, C. Pisac, and H. Elemara, "A review on prognostics and health monitoring of proton exchange membrane fuel cell," Renewable and Sustainable Energy Reviews, vol. 75, pp. 440-450, 2017.

[10] R. H. Lin, X. N. Xi, P. N. Wang, B. D. Wu, and S. M. Tian, "Review on hydrogen fuel cell condition monitoring and prediction methods," International Journal of Hydrogen Energy, vol. 44, 2018.

[11] M. Jouin, R. Gouriveau, D. Hissel, M. C. Péra, and N. Zerhouni, "Prognostics and health management of pemfc - state of the art and remaining challenges," International Journal of Hydrogen Energy, vol. 38, no. 35, pp. 15307-15317, 2013.

[12] M. Bressel, M. Hilairet, D. Hissel, and B. O. Bouamama, "Extended kalman filter for prognostic of proton exchange membrane fuel cell," Applied Energy, vol. 164, no. feb.15, pp. 220-227, 2016.

[13] J. Chen, D. Zhou, C. Lyu, and C. Lu, "A novel health indicator for pemfc state of health estimation and remaining useful life prediction," International Journal of Hydrogen Energy, vol. 42, no. 31, pp. 20230 $20238,2017$.

[14] M. Bressel, M. Hilairet, D. Hissel, and B. O. Bouamama, "Fuel cell remaining useful life prediction and uncertainty quantification under an automotive profile," in Conference of the IEEE Industrial Electronics Society, Conference Proceedings.

[15] M. Jouin, R. Gouriveau, D. Hissel, M.-C. Péra, and N. Zerhouni, "Prognostics of pem fuel cell in a particle filtering framework," International Journal of Hydrogen Energy, vol. 39, no. 1, pp. 481-494, 2014.
[16] M. Jouin, R. Gouriveau, D. Hissel, M. Pera, and N. Zerhouni, "Prognostics of proton exchange membrane fuel cell stacks in a particle filtering framework including characterization disturbances and voltage recovery," 2014.

[17] K. Chen, S. Laghrouche, and A. Djerdir, "Fuel cell health prognosis using unscented kalman filter: Postal fuel cell electric vehicles case study," International Journal of Hydrogen Energy, vol. 44, no. 3, pp. 1930-1939, 2019.

[18] Y. Wu, E. Breaz, F. Gao, and A. Miraoui, "A modified relevance vector machine for pem fuel cell stack aging prediction," IEEE Transactions on Industry Applications, vol. 52, no. 3, pp. 2573-2581, 2016.

[19] S. Kundu, M. Fowler, L. C. Simon, and R. Abouatallah, "Reversible and irreversible degradation in fuel cells during open circuit voltage durability testing," Journal of Power Sources, vol. 182, no. 1, pp. 254 258,2008

[20] S. Morando, S. Jemei, D. Hissel, R. Gouriveau, and N. Zerhouni, "Proton exchange membrane fuel cell ageing forecasting algorithm based on echo state network," International Journal of Hydrogen Energy, vol. 42 no. 2, pp. 1472-1480, 2016.

[21] Z. Hua, Z. Zheng, E. Pahon, M.-C. Péra, and F. Gao, "Remaining useful life prediction of pemfe systems under dynamic operating conditions," Energy Conversion and Management, vol. 231, p. 113825, 2021.

[22] Z. Li, Z. Zheng, and R. Outbib, "Adaptive prognostic of fuel cells by implementing ensemble echo state networks in time-varying model space," IEEE Transactions on Industrial Electronics, vol. 67, no. 1, pp. 379-389, 2020

[23] R. Ma, T. Yang, E. Breaz, Z. Li, P. Briois, and F. Gao, "Data-driven proton exchange membrane fuel cell degradation predication through deep learning method," Applied Energy, vol. 231, pp. 102-115, 2018.

[24] K. Chen, S. Laghrouche, and A. Djerdir, "Degradation prediction of proton exchange membrane fuel cell based on grey neural network model and particle swarm optimization," Energy Conversion and Management, vol. 195, pp. 810-818, 2019.

[25] R. E. Silva, R. Gouriveau, S. Jemei, D. Hissel, L. Boulon, K. Agbossou, and N. Y. Steiner, "Proton exchange membrane fuel cell degradation prediction based on adaptive neuro-fuzzy inference systems," International Journal of Hydrogen Energy, vol. 39, no. 21, pp. 11 128-11 144, 2014.

[26] Y. Cheng, N. Zerhouni, and C. Lu, "A hybrid remaining useful life prognostic method for proton exchange membrane fuel cell," International Journal of Hydrogen Energy, vol. 43, no. 27, pp. 12314-12 327, 2018.

[27] D. Zhou, F. Gao, E. Breaz, A. Ravey, and A. Miraoui, "Degradation prediction of pem fuel cell using a moving window based hybrid prognostic approach," Energy, vol. 138, no. nov.1, pp. 1175-1186, 2017.

[28] D. Zhang, "Contribution to prognostics of proton exchange membrane fuel cells : approaches based on degradation information at multiple levels," 2018.

[29] Ibrahim, Mona, Steiner, N. Yousfi, Jemei, Samir, Hissel, and Daniel, "Wavelet-based approach for online fuel cell remaining useful lifetime prediction," IEEE Transactions on Industrial Electronics, 2016.

[30] F. Yang, G. Enzner, and J. Yang, "Frequency-domain adaptive kalman filter with fast recovery of abrupt echo-path changes," IEEE Signal Processing Letters, vol. 24, no. 12, pp. 1778-1782, 2017.

[31] G. Bernardi, T. V. Waterschoot, J. Wouters, and M. Moonen, "Adaptive feedback cancellation using a partitioned-block frequency-domain kalman filter approach with pem-based signal prewhitening," IEEE/ACM Transactions on Audio Speech and Language Processing, vol. 25, no. 9, pp. 1480-1494, 2017.

[32] R. Gouriveau, M. Hilairet, D. Hissel, S. Jemei, M. Jouin, E. Lechartier, S. Morando, E. Pahon, M.-C. Pera, and N. Zerhouni, "Ieee phm 2014 data challenge: outline, experiments, scoring of results, winners." 2014

[33] J. K. Kimotho, T. Meyer, and W. Sextro, "Pem fuel cell prognostics using particle filter with model parameter adaptation," 2015.

[34] M. S. Jha, G. Dauphin-Tanguy, and B. Ould-Bouamama, "Particle filter based hybrid prognostics for health monitoring of uncertain systems in bond graph framework," Mechanical Systems and Signal Processing, pp. 301-329, 2016

[35] R. Lin, B. Li, Y. P. Hou, and J. M. Ma, "Investigation of dynamic driving cycle effect on performance degradation and micro-structure change of pem fuel cell," International Journal of Hydrogen Energy, vol. 34, no. 5, pp. 2369-2376, 2009.

[36] G. Enzner and P. Vary, "Frequency-domain adaptive kalman filter for acoustic echo control in hands-free telephones," Signal Processing, vol. 86, no. 6, pp. p. 1140-1156, 2006.

[37] W. Fan, K. Chen, J. Lu, and J. Tao, "Efficient improvement of frequencydomain kalman filter," 2018.

[38] H. Cherragui, M. Bressel, M. Hilairet, and S. Giurgea, "Fuel cells remaining useful life real-time estimation using an extended kalman 
filter in a hardware in the loop platform," in 2017 IEEE Vehicle Power and Propulsion Conference (VPPC), Conference Proceedings, pp. 1-6.

[39] Q. Zhu, M. Xu, W. Liu, and M. Zheng, "A state of charge estimation method for lithium-ion batteries based on fractional order adaptive extended kalman filter," Energy, vol. 187.

[40] A. Saxena, J. Celaya, B. Saha, S. Saha, and K. Goebel, "Metrics for offline evaluation of prognostic performance," International Journal of Prognostics and Health Management, vol. 1, pp. 2153-2648, 2010.

[41] D. Zhang, C. Cadet, C. Bérenguer, and N. Yousfi-Steiner, "Some improvements of particle filtering based prognosis for pem fuel cells," 2016.

[42] F. Yang, G. Enzner, and J. Yang, "Frequency-domain adaptive kalman filter with fast recovery of abrupt echo-path changes," IEEE Signal Processing Letters, vol. 24, no. 12, pp. 1778-1782, 2017. 